\title{
Quantum Fluctuations of Particles and Fields in Smooth Path Integrals
}

\author{
Takayasu SeKiHARA ${ }^{1}$ \\ ${ }^{1}$ KEK Theory Center, Institute of Particle and Nuclear Studies, High Energy Accelerator Research \\ Organization (KEK), 1-1, Oho, Tsukuba, Ibaraki, 305-0801, Japan \\ E-mail: sekihara@post.kek.jp
}

(Received July 14, 2013)

An approach to evaluation of the smooth Feynman path integrals is developed for the study of quantum fluctuations of particles and fields in Euclidean time-space. The paths are described by sum of Gauss functions and are weighted with $\exp (-S)$ by appropriate methods. The weighted smooth paths reproduce properties of the ground state of the harmonic oscillator in one dimension with high accuracy. Quantum fluctuations of U(1) and SU(2) gauge fields in four dimensions are also evaluated in our approach.

KEYWORDS: Path integrals, quantum mechanics, quantum field theory

\section{Introduction}

To clarify properties of the ground state of the system is one of the most important problems in quantum physics. For instance, the ground state of the one-dimensional harmonic oscillator fluctuates around the minimum of the potential due to the quantum effects in contrast to the classical situation, in which the oscillator keeps to stay at the minimum of the potential. We can "visualize" such quantum fluctuations by using the path integral method developed by Feynman [1], in which all possible paths are taken into account with the probability amplitude $\exp (-S)$ in Euclidean time-space, with $S$ the action of the system.

Although we cannot solve exactly the path integrals in almost all cases, the evaluation of the path integrals can be simplified by discretizing time-space, in which derivations and integrations are replaced with finite differences and summations, respectively, and measure of the path integrals becomes a countable product. This discretization, together with the weight $\exp (-S)$, allows us to describe easily the ground state of the system both in numerical and analytical calculations. An important example of the discretized path integrals is the lattice QCD (quantum chromodynamics) [2], by which nonperturbative aspects of QCD have been revealed [3].

However, the time-space discretization also has some shortcomings. Obviously, the time-space discretization explicitly breaks continuous symmetries of time-space such as the translational symmetry down to discrete symmetries. Furthermore, the discretization sometimes leads to qualitative discrepancies compared to the continuous theory such as the famous doubler for the fermions on the lattice [4] and magnetic monopoles in the lattice QED (quantum electrodynamics) [5]. Since they are brought by the time-space discretization, it is desired to perform the path integrals in smooth time-space from the viewpoint of complementarity for the discretized approach.

Motivated by these observations, we develop an approach to evaluating the smooth path integrals in Euclidean time for particles and fields [6]. The smooth paths are described by sum of Gauss functions with weight $\exp (-S)$ by appropriate methods such as the Metropolis method [7] and the heat-bath method [8]. 


\section{Basic ideas}

Firstly, for a nonrelativistic particle with one degree of freedom $q$ in a periodic boundary condition with period $\mathcal{T}$ in time direction, $q(\tau+\mathcal{T})=q(\tau)$, the path integral method evaluates the quantum transition amplitude in Euclidean time as [1],

$$
\mathcal{Z}=\int_{\text {period }} \mathcal{D} q \exp (-S[q]), \quad \mathcal{D} q \equiv \prod_{\tau} d q(\tau) .
$$

Since the quantum fluctuations of the particle are weighted with the factor $\exp (-S)$, an expectation value of an operator $O[q]$ in quantum mechanics can be evaluated by using $N$ paths $q_{n}$ ( $n=$ $1,2, \cdots, N)$ which are appropriately weighted with $\exp (-S)$ as,

$$
\langle O[q]\rangle=\frac{1}{\mathcal{Z}} \int_{\text {period }} \mathcal{D} q O[q] \exp (-S) \approx \frac{1}{N} \sum_{n=1}^{N} O\left[q_{n}\right],
$$

where the last approximation becomes good for large $N$.

Then, we consider the discretized approach to the path integrals for a nonrelativistic particle in the periodic boundary condition. In this case the particle position is represented as $\tilde{q}_{i}$ at time $\tilde{\tau}_{i}=i a$ with $i=1, \cdots, N_{\text {lat }}$ and the lattice spacing $a \equiv \mathcal{T} / N_{\text {lat }}$, and the path of the particle is obtained by connecting $\tilde{q}_{i}$ and $\tilde{q}_{i+1}$ from $i=1$ to $N_{\text {lat }}$ with straight lines. Then measure of the path integral is now defined as a countable product and the action is replaced with the corresponding discretized one, $\tilde{S}[\tilde{q}]$. The details of the discretized path integrals for nonrelativistic particles are discussed in Ref. [9].

Now let us make our procedure for the simulation of the smooth path integrals from analogy to the discretized approach. Our idea is to connect the neighboring points for the particle positions by smooth lines rather than straight lines. In order to achieve this, we smear the particle position in the discretized approach $\tilde{q}_{i}$ with a Gauss function of width $\xi_{i}$ at time $\tau_{i}$,

$$
\tilde{q}_{i}\left(\text { at } \tau=\tilde{\tau}_{i}\right) \rightarrow q_{i} \exp \left[-\frac{\left(\tau-\tau_{i}\right)^{2}}{\xi_{i}^{2}}\right],
$$

for $i=1$ to $N_{\text {lat }}$, where $\tau-\tau_{i}$ means to take time distance between $\tau$ and $\tau_{i}$ in the periodic boundary condition. Here $\left(q_{i}, \tau_{i}, \xi_{i}\right)$ in our approach corresponds to $\left(\tilde{q}_{i}, \tilde{\tau}_{i}, a\right)$ in the discretized approach. With this smearing one can naturally connect the path with smooth lines rather than straight lines as,

$$
q(\tau)=\sum_{i=1}^{N_{\text {sum }}} q_{i} \exp \left[-\frac{\left(\tau-\tau_{i}\right)^{2}}{\xi_{i}^{2}}\right]
$$

where $N_{\text {sum }}\left(=N_{\text {lat }}\right)$ is number of the summed terms. In this construction of the smooth path the minimal scale of the fluctuation for $q(\tau)$ corresponds to $\xi_{i}$. The positions of the Gauss functions $\tau_{i}$ may take random values rather than values in same interval, $\tau_{i}=i \mathcal{T} / N_{\text {sum }}$, as long as every time is equally treated without making any special time. The width of the Gauss function, or the scaling constant, $\xi_{i}$, should be determined so as to prevent any special time coordinate. In this study we use a properly fixed value for $\xi$. Then the weight $\exp (-S)$ is given by appropriate methods for $q_{i}$ as in the discretized approach [9]. If the action $S[q]$ is expressed as polynomials of $q(\tau)$ and $\dot{q}(\tau) \equiv d q / d \tau$, the action can be rewritten as polynomials of $q_{i}$ due to the expression of $q(\tau)$ in Eq. (4) and properties of the Gauss functions, and hence we can perform the heat-bath method [8] for the weight $\exp \left[-S\left(q_{i}\right)\right]$ with respect to $q_{i}$. If the action $S[q]$ is not expressed as polynomials of $q_{i}$, one may use the Metropolis method [7] by considering the charge $\delta q_{i}$ with respect to $q_{i}$ and the corresponding change of the action $S\left(q_{i}\right)-S\left(q_{i}+\delta q_{i}\right)$. The update of $q_{i}$ is performed until the action converges. 
Here we note that $q(\tau)$ in Eq. (4) cannot describe all possible paths, since the Gauss functions in Eq. (4) cannot be a complete set with respect to the smooth functions in the periodic boundary condition. Therefore, at this point our construction of paths is an approximation with respect to the complete paths required by the measure $\mathcal{D} q$. Nevertheless, we expect that most of the possible paths can be effectively taken into account when value of $N_{\text {sum }}$ is sufficiently large with dense $\tau_{i}$ and small $\xi$. We also note that time uniformity may be broken when the time components $\tau_{i}$ take values in same interval, $\tau_{i}=i \mathcal{T} / N_{\text {sum }}$, but we expect that the uniformity will restore if one considers sufficiently dense distribution of the Gauss functions. Generalization to the systems with $f$ degrees of freedom $(f \geq 2)$ as well as to quantum field theories is obvious.

\section{Numerical results}

Now let us examine our approach by investigating a harmonic oscillator in one dimension, which action is written as,

$$
S_{\mathrm{HO}}=\int_{0}^{\mathcal{T}} d \tau L_{\mathrm{HO}}(q, \dot{q}), \quad L_{\mathrm{HO}}=\frac{1}{2} m \dot{q}^{2}+\frac{1}{2} m \omega^{2} q^{2} .
$$

Here we fix its mass and angular frequency as $m=\omega=1$ and take the time range $\mathcal{T}=20$. We consider four simulation conditions; $\xi=2.0$ (A), $\xi=1.0(\mathrm{~B}), \xi=0.5$ (C), and $\xi=0.1$ (D), with the same number of the summation $N_{\text {sum }}=200$ in each condition. For the initial condition we fix $\tau_{i}$ as $\tau_{i}=i \mathcal{T} / N_{\text {sum }}$ and randomly generate $q_{i}$ as a hot start. We prepare $N=400$ paths for each condition. Since temperature of the system $1 / \mathcal{T}$ is much smaller than the excitation energy, the quantum fluctuations in this study will reflect the ground state of the harmonic oscillator.

As a result of the numerical simulation, quantum paths approach to equilibrium at around the iteration number $N_{\text {iteration }} \sim 5 \times 10^{4}$ for all conditions. After iteration $N_{\text {iteration }}=5 \times 10^{4}$ the expectation value of the mean squared radius of the fluctuation becomes $\left\langle\overline{q^{2}}\right\rangle=0.389 \pm 0.08$ for the condition $\mathrm{A}$, $0.433 \pm 0.008$ for $\mathrm{B}$, and $0.464 \pm 0.008$ for $\mathrm{C}$, and $0.488 \pm 0.008$ for $\mathrm{D}$, where we used an average form $\overline{q^{2}} \equiv \int_{0}^{\mathcal{T}} d \tau q(\tau)^{2} / \mathcal{T}$. The expectation value of the mean squared radius approaches to the ground-state value 0.5 as the scale constant $\xi$ gets small and hence structures of the quantum fluctuations in fine scale can be described, which is similar to the case of the discretized approach. Then let us visualize degree of the quantum fluctuations by making a histogram for $q$ with division of time range into
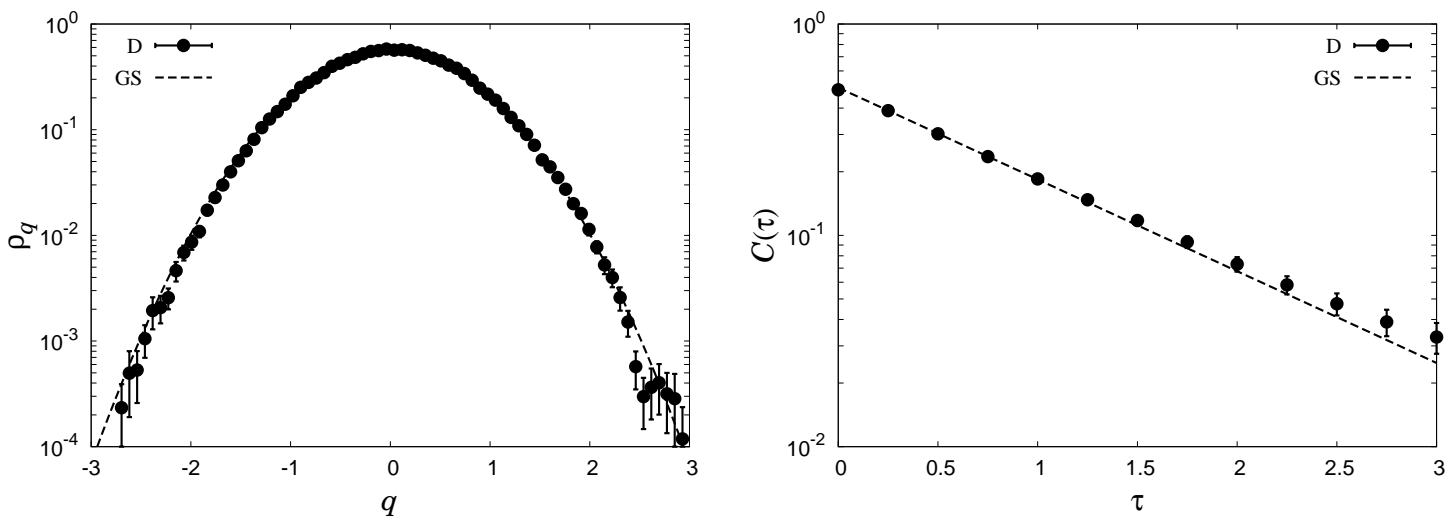

Fig. 1. The distribution of the coordinate for the harmonic oscillator in condition $\mathrm{D}$ ( $\xi=0.1$ and $\left.N_{\mathrm{sum}}=200\right)$ together with the squared wave function of the ground state denoted by dashed line (left) and the correlation function for the fluctuation in condition $\mathrm{D}$ together with the exact value for the ground state denoted by dashed line (right). Both are given in logarithmic scale. 
sufficiently many parts in each path and then combine $N=400$ histograms to obtain the $q$-distribution $\rho_{q}$. The result for the condition D is shown in Fig. 1(left) together with the squared wave function of the ground state. As one can see, our $q$-distributions in the condition D behave consistently with the squared wave function. Especially it is interesting that behavior of the quantum fluctuations to large $q(\sim \pm 3)$ is very similar to the squared wave function. Furthermore, from the fluctuation one can evaluate the correlation function defined as $C(\tau) \equiv\langle\overline{q(\tau) q(0)}\rangle=\int_{0}^{\mathcal{T}} d \tau^{\prime} q\left(\tau+\tau^{\prime}\right) q\left(\tau^{\prime}\right) / \mathcal{T}$. The correlation function in the condition $\mathrm{D}$ is shown in Fig. 1(right) together with the exact value for the grond state $=0.5 \times \exp (-\tau)$. As one can see, the correlation function in condition D is quite similar to the exact value up to $\tau \sim 2$ and the behavior is consistent even in larger $\tau$.

In the examination of our approach for the harmonic oscillator, we have seen that our approach reproduces quantum behavior of the system with more than about $95 \%$ accuracy by properly chosen scale constants. Especially, one can obtain higher accuracy by using smaller scaling constant $\xi$.

Our approach can be applied to relativistic field theories. It is important that our approach has possibilities to become a nonperturbative way to quantum field theories. To be specific, we simulate $\mathrm{U}(1)$ and $\mathrm{SU}(2)$ gauge fields in four dimensions with a periodic boundary condition (for the detail of the simulation condition, see Ref. [6]). In this study we do not include gauge fixing terms nor the Faddeev-Popov ghosts in the Lagrangian densities. Although the gauge invariance is maintained if one takes into account all the possible paths for gauge fields, this is not the case in our approach with finite $N_{\text {sum }}$. Nevertheless, the gauge group manifold is expected to be effectively taken into account if one takes sufficiently large $N_{\text {sum }}$ with small $\xi$ in our approach. One of the interesting results that at the saturation point $\left\langle\overline{\mathcal{L}}_{\mathrm{SU}(2)}\right\rangle \approx 0.49 \xi^{-4}$ is smaller than $3 \times\left\langle\overline{\mathcal{L}}_{\mathrm{U}(1)}\right\rangle \approx 3 \times 0.20 \xi^{-4}$ due to the self-interactions in $\operatorname{SU}(2)$, where $\overline{\mathcal{L}}$ is averaged Lagrangian density $\left[\overline{\mathcal{L}} \equiv \int d^{4} x \mathcal{L}(x) / \mathcal{V}_{4}\right.$ with the four dimensional volume $\mathcal{V}_{4}$ ]. Furthermore, from the Wilson loop [2], we can evaluate a potential between the (infinitely heavy) fundamental representation and its antiparticle, which results in the Coulomb force and a confining linear potential for the $\mathrm{U}(1)$ and $\mathrm{SU}(2)$ gauge fields, respectively.

\section{Summary}

In summary, we have developed an approach to evaluation of the smooth path integrals, in which paths are described by sum of smooth functions with weight $\exp (-S)$ by appropriate methods. In this study we take an approximation that smooth fluctuations are described only by the Gauss function. The weighted smooth paths reproduce properties of the ground-state harmonic oscillator in one dimension with high accuracy by properly chosen width of the Gauss functions, and the accuracy gets higher by using smaller width of the Gauss functions, with which finer structure of the quantum fluctuations can be described. We have also evaluated quantum fluctuations of the U(1) and SU(2) gauge fields in four dimensions and the Coulomb force and a confining linear potential have been extracted from the $\mathrm{U}(1)$ and $\mathrm{SU}(2)$ gauge fields, respectively.

\section{References}

[1] R. P. Feynman, Rev. Mod. Phys. 20 (1948) 367.

[2] K. G. Wilson, Phys. Rev. D 10 (1974) 2445.

[3] H. J. Rothe, Lattice Gauge Theories: An Introduction (World Scientific, 2005).

[4] K. G. Wilson, Quarks and Stings on a Lattice, in "Gauge Theories and Modern Field Theory" (MIT Press, Cambridge, 1975).

[5] A. M. Polyakov, Phys. Lett. B 59 (1975) 82.

[6] T. Sekihara, arXiv:1201.0055 [quant-ph].

[7] N. Metropolis, A. W. Rosenbluth, M. N. Rosenbluth, A. H. Teller and E. Teller, J. Chem. Phys. 21 (1953) 1087.

[8] M. Creutz, Phys. Rev. D 21 (1980) 2308.

[9] M. Creutz and B. Freedman, Annals Phys. 132 (1981) 427. 